\title{
ON COUNTABLY GENERATED $z$-IDEALS OF $C(X)$ FOR FIRST COUNTABLE SPACES
}

\author{
ATTILIO LE DONNE
}

\begin{abstract}
In paper [L], a question asked in [D] has been answered first by proving that: if $X$ is normal and first countable, then every countably generated $z$-ideal of $C(X)$ is pure; then, by giving an example of a nonpure countably generated $z$-ideal of $C(X)$ in a $\sigma$-compact (hence normal) but not first countable space $X$.

In this paper a class $\mathcal{C}$ of topological spaces $X$ whose $C(X)$ has a nonpure countably generated $z$-ideal is constructed; it is proved that $\mathcal{C}$ contains a space $X$ which is first countable. So it is proved that in the proposition above the hypotheses "normal" and "first countable" are both essential.

Finally in $84 \mathrm{I}$ prove, as announced in [L], that if $X$ is a locally compact normal space, then every countably generated $z$-ideal of $C(X)$ is pure.

For the terminology and notations see [GJ], [D], [L].
\end{abstract}

1. The classes $\mathscr{B}$ and $\mathcal{C}$. Let $\mathscr{B}$ be the class of topological spaces $B$ which are disjoint unions (but not topological sums) of three subspaces $C, D, E$, with the properties:

$\left(\mathrm{P}^{\mathrm{I}}\right) C=\omega \times D(\omega$ is the discrete space of natural numbers);

$\left(\mathrm{P}^{\mathrm{II}}\right)$ there exists $g: B \rightarrow R$ continuous such that $Z(g)=D$ and $g$ is constant on the sets $\{n\} \times D \subset C$ for each $n \in \omega$;

$\left(\mathrm{P}^{\mathrm{II}}\right)$ if $f: B \rightarrow R$ is continuous and vanishes for every $n \in \omega$ on a point $\left(n, d_{n}\right) \in C$ then $f$ vanishes at some point of $D$;

$\left(P^{I V}\right) D \subset$ int $_{B}(B \backslash C)$.

Fix a bijection $u$ : $\omega \rightarrow \omega \times \omega, u(s)=\left(s_{1}, s_{2}\right)$. For each $B \in \mathscr{B}$ let

$$
\Lambda(B)=B \times \omega \times \omega .
$$

Let $\mu: \Lambda(B) \rightarrow \Lambda(B)$ be the (noncontinuous) map which is the identity on $(B \backslash D) \times \omega \times \omega$ and maps the point $x=(d, n, m) \in D \times \omega \times \omega$ into the point $\mu(x)=\left(\left(n_{1}, d\right), n_{2}, m+1\right) \in C \times \omega \times \omega$. Clearly $\mu$ is idempotent (i.e., $\left.\mu \circ \mu=\mu\right)$.

We define $X(B)$ as the quotient of $\Lambda(B)$ by the equivalence relation which identifies $x$ and $\mu(x)$, for every $x \in \Lambda(B)$.

The spaces $X(B)$ with $B$ running over $\mathscr{B}$ form the class $C$.

We note that the class $\mathscr{B}$ (and hence the class $\mathcal{C}$ ) is nonvoid: in fact, the "book" $B$ of [L] satisfies $\left(\mathrm{P}^{\mathrm{I}}\right)-\left(\mathrm{P}^{\mathrm{IV}}\right)$ with $C$ the "top section", $D$ the "back"; moreover $X(B)$ for such a "book" $B$ is exactly the space $X$ of $[L]$.

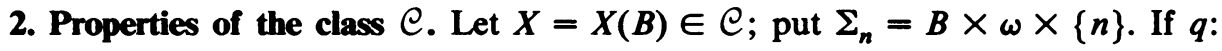
$\Lambda(B) \rightarrow X(B)$ is the quotient map, put $Z_{n}=q\left(\cup_{k>n+1} \Sigma_{k}\right)$.

Received by the editors April 15, 1980 and, in revised form, July 14, 1980. 1980 Mathematics Subject Classification. Primary 54C40; Secondary 54G20. 
LEMMA 2.1. $Z_{n}$ is a zeroset of $X$.

The proof is analogous to the construction of the sequence $\left(f_{n}\right)$ of $[\mathbf{L}, 2.2]$, using the existence of the function $g$ of property $\left(\mathrm{P}^{\mathrm{II}}\right)$.

LEMMA 2.2. $Z_{n+1} \subset$ int $_{X} Z_{n}$.

Proof. By property $\left(\mathrm{P}^{\mathrm{IV}}\right)$ the set $D \times \omega \times\{n\}$ is contained in

$$
\operatorname{int}_{\Lambda}((B \backslash C) \times \omega \times\{n\}),
$$

then

$$
\left(\bigcup_{k>n+1} \Sigma_{k}\right) \cup(D \times \omega \times\{n\})
$$

is contained in the interior of

$$
\left(\bigcup_{k>n+1} \Sigma_{k}\right) \cup((B \backslash C) \times \omega \times\{n\})
$$

and these two sets are $q \leftarrow \circ q$ stable.

LEMMA 2.3. Every continuous function $f \in C(X)$ vanishing on $X \backslash Z_{1}$ vanishes at some point of $Z_{n}$.

Proof. The function $f \circ q: \Lambda \rightarrow \mathbf{R}$ vanishes at every point of $C \times \omega \times\{1\}$. Then by property $\left(\mathrm{P}^{\mathrm{III}}\right)$ it vanishes at a point of $D \times\{k\} \times\{1\}$ for every $k \in \omega$. By the equivalence relation, $f \circ q$ vanishes at a point $\left(\left(n, d_{n}^{(h)}\right), h, 2\right) \in C \times\{h\} \times\{2\}$ for every $h \in \omega$; then by $\left(\mathrm{P}^{\mathrm{III}}\right)$ it vanishes at a point of $D \times\{h\} \times\{2\}$; and so on.

Then by Lemma 1.2 of [L], we have

Proposition 2.1. If $X \in \mathcal{C}$ then $C(X)$ has a nompure countably generated $z$-ideal.

3. Construction of a $B \in \mathscr{B}$ such that $X(B)$ is a $T_{3 \frac{1}{2}}$ (also zero-dimensional) first countable space. We put $D=\omega^{\omega}, C=\omega \times D$ and $E=\omega \times \omega \times D \times D$; and $B=(\omega \times \omega \times D \times D) \cup(\omega \times D) \cup D$ with the topology given by:

(I) the points of $E$ are isolated;

(II) the points $x=(n, p) \in \omega \times D$ have as a countable neighbourhood base the sets

$$
\{x\} \cup(\{h \mid h>k\} \times\{n\} \times D \times\{p\})
$$

with $k$ running over $\omega$;

(III) the points $p=\left(m_{h}\right)_{h \in \omega} \in D$ have as a countable neighbourhood base the sets

$$
\{p\} \cup\left(\left\{\left(m_{h}, h\right) \mid h>k\right\} \times\{p\} \times D\right)
$$

with $k$ running over $\omega$.

It may be verified that each countable neighbourhood base, as given, consists of clopen sets; hence $B$ is a $T_{2}$ zero-dimensional first countable space.

LEMMA 3.1. $B \in \mathscr{B}$.

Proof. Properties $\left(\mathbf{P}^{\mathrm{I}}\right)$ and $\left(\mathrm{P}^{\mathrm{IV}}\right)$ are obvious.

Property $\left(\mathrm{P}^{\mathrm{II}}\right)$. Define $\phi: B \rightarrow R$ to be zero on $D$ and put $\phi(m, n, p, q)=2^{-n}$ and $\phi(n, p)=2^{-n}$. 
Property ( $\left.\mathrm{P}^{\mathrm{III}}\right)$. Let $f: B \rightarrow R$ be continuous and let $p_{n} \in D$ such that $f\left(n, p_{n}\right)=0$. Then there exists $k_{n}$ such that on the set $\left\{k_{n}\right\} \times\{n\} \times D \times\left\{p_{n}\right\}$ the function $|f|$ is smaller than $1 / n$. Now if $p=\left(k_{n}\right)_{n}$ we have $f(p)=0$.

As we have said in $\S 2, X(B)$ consists of the equivalence classes $\{n, \mu(x)\}$ of $\Lambda(B)$ with $x$ running over $(E \cup D) \times \omega \times \omega$.

If $x \in E \times \omega \times \omega$, we have $x=\mu(x)$ and the point $x$ is isolated in $\Lambda(B)$ and so in $X(B)$. If $x \in D \times \omega \times \omega$ we can take the union of a neighbourhood of type (III) of $x$ with one of type (II) of $\mu(x)$; this union is clopen in $\Lambda(B)$ and $q^{\leftarrow} \circ q$ stable: then also $q(x)$ has clopen neighbourhood base in $X(B)$. If $x \in C \times \omega \times\{1\}$, we have $\mu(x)=x$ and it suffices to take the neighbourhood base of $x$ of type (III).

This proves that $X(B)$ is a first countable Hausdorff zero-dimensional space.

\section{Locally compact normal space.}

LEMMA 4.1. If $X$ is locally compact, then every countably generated $z$-ideal I of $C(X)$ has a sequence of generators $f_{0}, f_{1}, \ldots$ such that int $_{X} Z\left(f_{n}\right) \supset Z\left(f_{n+1}\right)$ for every $n \in N$.

The proof is analogous to that of Lemma 1.5 of $[\mathbf{L}]$. Let $g_{0}, g_{1}, \ldots$ be a sequence of generators of $I$ with $\left(g_{0}\right) \subset\left(g_{1}\right) \subset \ldots$ and $g_{0}<g_{1}<\ldots$. Assume that int $_{X} Z\left(g_{0}\right)$ fails to contain any $Z\left(g_{n}\right)$; then for each $n$ there exists a $p_{n} \in \operatorname{cl}_{X} \operatorname{coz}\left(g_{0}\right)$ $\cap Z\left(g_{n}\right)$. Let $V_{n}$ be a compact neighbourhood of $p_{n}$ in $X$. There is a sequence $x_{m}^{n} \in V_{n}$ with $0 \neq\left|g_{0}\left(x_{m}^{n}\right)\right|<\left|g_{n}\left(x_{m}^{n}\right)\right|<1 / m . B_{n}=\operatorname{cl}_{X}\left\{x_{m}^{n}: m \in N\right\}$ is compact. Besides $B_{n} \backslash\left\{x_{m}^{n}: m \in N\right\}$ is not void and is contained in $Z\left(g_{n}\right)$. Now $S_{n}=$ $\operatorname{cl}_{\beta X} Z\left(g_{0}\right) \cup B_{n}$ is a closed subset of $\beta X$; the function which is zero on $\operatorname{cl}_{\beta X} Z\left(g_{0}\right)$ and is $\left|g_{n}\left(x_{m}^{n}\right)\right|^{1 / 2}$ on $\left\{x_{m}^{n}: m \in N\right\}$ is continuous on $S_{n}$; hence it has a continuous extension $h_{n}^{*} \in C(\beta X)$. Let $h_{n}=h_{n}^{*} \mid X$. Put $h=\Sigma_{n=0}^{\infty}\left(h_{n} \wedge 2^{-n}\right)$; by uniform convergence $h \in C(X)$. Clearly $Z(h) \supset Z\left(g_{0}\right)$; but $h$ cannot be a multiple (in $C(X)$ ) of any $g_{n}$. For if $h=u g_{k}$, as in [L, Lemma 1.5] we see that $u$ cannot be continuous at any point of $B_{n} \backslash\left\{x_{m}^{n}: m \in N\right\}$.

Then by $[\mathrm{L}, 1.3(\mathrm{ii})]$ we have

Proposition 4.2. If $X$ is normal and locally compact, then every countably generated $z$-ideal of $C(X)$ is pure.

\section{REFERENCES}

[D] G. De Marco, On the countably generated z-ideals of $C(X)$, Proc. Amer. Math. Soc. 31 (1972), 574-576.

[GJ] L. Gillman and M. Jerison, Rings of continuous functions, Van Nostrand, New York, 1960.

[L] A. Le Donne, On a question concerning countably generated z-ideals of $C(X)$, Proc. Amer. Math. Soc. 80 (1980), 505-510.

Seminario Matematico, Università di Padova, via Belzoni 7, 35100 Padova, Italy 